\title{
Development of a Novel Cell Surface Attachment System to Display Multi-Protein Complex Using the Cohesin-Dockerin Binding Pair
}

\author{
Hyeok-Jin $\mathrm{Ko}^{1 \dagger}$, Heesang Song ${ }^{2 \dagger}$, and In-Geol Choi ${ }^{3 *}$ \\ ${ }^{1}$ Food Biotech R\&D Center, Samyang Corp., Seongnam 13488, Republic of Korea \\ ${ }^{2}$ Department of Biochemistry and Molecular Biology, Chosun University School of Medicine, Gwangju 61452, \\ Republic of Korea \\ ${ }^{3}$ Department of Biotechnology, College of Life Sciences and Biotechnology, Korea University, Seoul 02841, Republic \\ of Korea
}

\begin{abstract}
Autodisplay of a multimeric protein complex on a cell surface is limited by intrinsic factors such as the types and orientations of anchor modules. Moreover, improper folding of proteins to be displayed often hinders functional cell surface display. While overcoming these drawbacks, we ultimately extended the applicability of the autodisplay platform to the display of a protein complex. We designed and constructed a cell surface attachment (CSA) system that uses a noncovalent protein-protein interaction. We employed the high-affinity interaction mediated by an orthogonal cohesin-dockerin (Coh-Doc) pair from Archaeoglobus fulgidus to build the CSA system. Then, we validated the orthogonal Coh-Doc binding by attaching a monomeric red fluorescent protein to the cell surface. In addition, we evaluated the functional anchoring of proteins fused with the Doc module to the autodisplayed Coh module on the surface of Escherichia coli. The designed CSA system was applied to create a functional attachment of dimeric a-neoagarobiose hydrolase to the surface of $E$. coli cells.
\end{abstract}

Keywords: Escherichia coli cell surface attachment, non-covalent interaction module, cohesin-dockerin, aneoagarobiose hydrolase

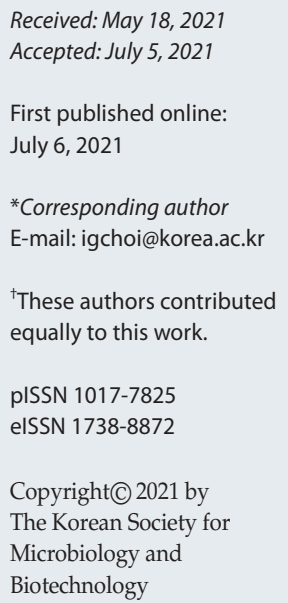

\section{Introduction}

Microbial cell surface display (CSD) is regarded as a powerful tool in various biotechnological applications [1]. Many techniques have been developed using an assortment of anchoring proteins, such as outer membrane proteins, lipoproteins, subunits of surface appendages (e.g., flagella and pilus), or S-layer proteins to display proteins of interest (POIs) on the surfaces of bacteria [2]. However, many critical factors can limit the functional and active display of POIs. For instance, the size and structure of POIs to be displayed can cause low-level display at the cell surface and their overexpression can lead to host cell toxicity [3]. Among many approaches undertaken to overcome these limitations, "autodisplay" using autotransporters as the anchoring scaffold has been one of the most promising techniques because of lower levels of cellular toxicity and the ability to incorporate POIs of various sizes [3].

Although autodisplay platforms provide many attractive features for the CSD system, constraints still exist that limit the versatility of displayed proteins [4]. One such constraint is the retention of the functionality of proteins displayed in a multimeric form. A few reports have described the functional display of proteins having a dimeric active form by spontaneous dimerization within the limited space of the cell surface $[5,6]$. Because spontaneous dimerization can only occur between monomers in close proximity, this strategy can only be used when displayed proteins are expressed in high numbers, rendering this approach highly conditional. In some cases, there is a possibility of displayed proteins acquiring an inactive conformation due to improper folding [7].

To develop an alternative approach that can overcome these limitations, we designed a cell surface attachment (CSA) system in which POIs can be non-covalently attached to surface-displayed anchoring scaffolds. For noncovalent attachment, we searched protein families to select interacting protein domains that show specific binding interactions. We selected the cohesin-dockerin (Coh-Doc) domain pair to facilitate cell surface anchoring. The Coh-Doc system was originally discovered in many cellulolytic anaerobic bacteria where it is responsible for building extracellular macromolecular complexes such as the "cellulosome." The Coh-Doc system controls and 
maximizes the degradation efficiency of recalcitrant cellulose using a limited amount of enzymes [8]. The interaction between the Coh and Doc domains exhibits a very high binding specificity and strong affinity in a $\mathrm{Ca}^{2+}$-dependent manner $[9,10]$. Because Coh-Doc binding is known to be one of the strongest protein-protein interactions $\left(\mathrm{Kd}, 10^{-9}-10^{-11}\right)$ [11-14], the system has high potential as a biorecognition module [15].

We applied the Coh and Doc modules to the autodisplay platform and designed a CSA system to allow the functional expression of multi-complex proteins by non-covalent anchoring of heterologous proteins. To confer high orthogonality of applied modules, we surveyed potential Coh-Doc pairs predicted in various bacteria and selected the Coh-Doc pair from Archaeoglobus fulgidus [16]. Functional attachment was validated by a known dimeric enzyme ( $\alpha$-neoagarobiose hydrolase, NABH) that had previously failed to display functionally in the autodisplay platform [17]. In this study, we constructed a novel CSA system using Coh-Doc pairs as biorecognition modules and provided a promising tool for the extension of autodisplay platform applications.

\section{Materials and Methods}

\section{Bacterial Strains, Plasmids, and Culture Conditions}

Escherichia coli DH5a and E. coli BL21(DE3) were used as host strains for general cloning and recombinant protein overexpression, respectively. E. coli BW25113 was used for surface display of the Coh or Doc module. All E. coli cultures were aerobically grown in Luria-Bertani (LB) medium (Difco, USA) supplemented with $100 \mu \mathrm{g} / \mathrm{ml}$ ampicillin as the selectable marker. All E. coli competent cells were prepared by the TSS method [18]. Saccharophagus degradans $2-40^{\mathrm{T}}$ (ATCC 43961) was cultivated in a sea salt minimal medium as described by Ekborg et al. [19]. The genes encoding the Coh (Accession No. O30294) and Doc (Accession No. O30295) domains of A. fulgidus DSM 4304 were synthesized and codon optimized for E. coli from GeneArt (hereafter referred to as AfuCoh76 and AfuDoc75, respectively). The bacterial strains, plasmids, and primers for PCR are listed in Table 1.

Table 1. Bacterial strains, plasmids, and oligonucleotide primers used in this study.

\begin{tabular}{|c|c|c|}
\hline $\begin{array}{l}\text { Strain, plasmid, or } \\
\text { oligonucleotide }\end{array}$ & Relevant characteristic(s), description, or sequence ${ }^{a}$ & $\begin{array}{l}\text { Source or } \\
\text { reference }\end{array}$ \\
\hline \multicolumn{3}{|l|}{ Strains } \\
\hline E. coli DH5a & $\begin{array}{l}\mathrm{F}^{-} \text {endA1 glnV44 thi-1 recA1 relA1 gyrA96 deoR nup G } \Phi 80 d l a c Z \Delta M 15 \Delta \text { (lacZYA- } \\
\operatorname{argF}) U 169 \mathrm{hsdR} 17\left(r_{K}^{-} m_{K}^{+}\right) \lambda-\end{array}$ & Invitrogen \\
\hline E. coli BW25113 & F- $\Delta\left(\right.$ araD-araB) $567 \Delta l a c Z 4787(:: r r n B-3) \lambda^{-} r p h-1 \Delta(r h a D-r h a B) 568 h s d R 514$ & CGSC $^{\mathrm{a}}$ \\
\hline E. coli $\mathrm{BL} 21(\mathrm{DE} 3)$ & $\mathrm{F}-$ omp $\mathrm{T} h s d \mathrm{SB}(\mathrm{rB}-, \mathrm{mB}-)$ gal dcm (DE3) & Invitrogen \\
\hline S. degradans $2-40$ & Source of agarase genes and NABH & ATCC $^{b}$ \\
\hline \multicolumn{3}{|l|}{ Plasmids } \\
\hline AfuCoh76 & The plasmid carrying $A f u C o h 76$ gene synthesized using GeneArt & This study \\
\hline AfuDoc75 & The plasmid carrying AfuDoc75 gene synthesized using GeneArt & This study \\
\hline pJL vector & Derivative of pET21a vector; insertion of LIC sequence & $\begin{array}{l}\text { (Lee and } \\
\text { Kim, 2009) }\end{array}$ \\
\hline pJL-mRFP1: AfuCoh76 & pJL carrying the fusion gene comprised AfuCoh76 fused to the C-terminus of mRFP1 & This study \\
\hline pJL-mRFP1: AfuDoc75 & pJL carrying the fusion gene comprised AfuDoc75 fused to the C-terminus of mRFP1 & This study \\
\hline pJL-NABH: AfuDoc75 & pJL carrying the fusion gene comprised AfuDoc75 fused to the C-terminus of $\mathrm{NABH}$ & This study \\
\hline pJL-AfuDoc75: NABH & pJL carrying the fusion gene comprised AfuDoc75 fused to the N-terminus of NABH & This study \\
\hline pATLIC vector & Autodisplay vector based on the YfaL autotransporter & $\begin{array}{l}\text { (Ko et al. } \\
\text { 2012) }\end{array}$ \\
\hline pATLIC-AfuCoh76 & pATLIC carr & This study \\
\hline pATLIC-AfuDoc75 & pATLIC carrying the AfuDoc75 gene & This study \\
\hline \multicolumn{3}{|l|}{ Primers } \\
\hline AfCoh76-mRFP1_F & GTCGTCACTCCACCGGTGCTGCTAGTGCTGAAATGGTGGTTA & This study \\
\hline AfCoh76-pJL_R & ATGATGGTGATGGTGACCAGCACCACCTTTTACGG & This study \\
\hline AfDoc75-mRFP1_F & GTCGTCACTCCACCGGTGCTGAAGAAGCGAACAAAGGCGAC & This study \\
\hline AfDoc75-pJL_R & ATGATGGTGATGGTGAGGCCTTTTGCCCAGCAGGCCATTCT & This study \\
\hline mRFP1_F & GAAGGAGATATAAGGATGGCTTCCTCCGAAGACGTTATC & This study \\
\hline mRFP1_R & ATGATGGTGATGGTGAGCACCGGTGGAGTGACG & This study \\
\hline mRFP1-AfCoh76_R & ACCACCATTTCAGCACTAGCAGCACCGGTGGAGTGACG & This study \\
\hline mRFP1-AfDoc75_R & TCGCCTTTGTTCGCTTCTTCAGCACCGGTGGAGTGACG & This study \\
\hline AfCoh76at_F & CGGTGTCGCGCCCGCTAGTGCTGAAATGGTGGTTA & This study \\
\hline AfCoh76at_R & CGGTCGTTGGCCCACCAGCACCACCTTTTACGG & This study \\
\hline pATLIC $_{29 \_}$R & GTTGGCCCGGGCGCGACACCGTTAGCAGAGAAAA & This study \\
\hline AfDoc75at_F & CGGTGTCGCGCCCGAAGAAGCGAACAAAGGCGAC & This study \\
\hline AfDoc75at_R & CGGTCGTTGGCCCTTTGCCCAGCAGGCCATTCT & This study \\
\hline AfDoc75-pJL_F & GAAGGAGATATAAGGATGGAAGAAGCGAACAAAGGCGAC & This study \\
\hline AFDoc75-NABH_R & TTATTTACTTTTGAATCGCTTTTGCCCAGCAGGCCATTCTGAG & This study \\
\hline NABH-AfDoc75_F & AGAATGGCCTGCTGGGCAAAAGCGATTCAAAAGTAAATAAAAAATTGAG & This study \\
\hline NABH-pJL_R & ATGATGGTGATGGTGTACTGCTCCGGAATCGCCTGTTTG & This study \\
\hline
\end{tabular}

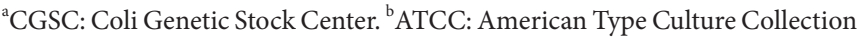


Construction and Cloning of Monomeric Red Fluorescent Protein (mRFP1) and NABH Tagged with AfuCoh76 and AfuDoc75 Modules

The gene sequences of AfuCoh76, AfuDoc75, mRFP1 [20], and NABH [21] were amplified by PCR using $\alpha$-Taq polymerase (GeneAll, Korea). To fuse mRFP1 to AfuCoh76 and AfuDoc75, overlap extension PCR (OE-PCR) was performed [22]. For the overexpression of fusion proteins, the resulting PCR products were cloned into a pJL vector having a 6xHis-tag at the C-terminus for affinity chromatography purification (pJL-mRFP1:AfuCoh76, mRFP1:AfuDoc75, -NABH:AfuDoc75, and -AfuDoc75:NABH; the position of AfuCoh76 and AfuDoc75 in the fusion proteins indicates the location at either the C-or the N-terminus) [23]. To autodisplay AfuCoh76 and AfuDoc75, the resulting PCR products were cloned into a pATLIC vector (pATLIC-AfuCoh76 and -AfuDoc75) [17]. All recombinant clones were confirmed by DNA sequencing.

\section{Overexpression and Purification of mRFP1:AfuCoh76 and mRFP1:AfuDoc75}

To purify mRFP1 tagged with either AfuCoh76 or AfuDoc75, E. coli BL21(DE3) cells having the designated plasmids were grown in $100 \mathrm{ml}$ of LB media with ampicillin $(100 \mu \mathrm{g} / \mathrm{ml})$ at $37^{\circ} \mathrm{C}$ and $200 \mathrm{rpm}$ to an $\mathrm{OD}_{600}$ of 0.8 and added to a final concentration of $0.5 \mathrm{mM}$ isopropyl thio- $\beta$-D-galactoside, and the cells were induced at $37^{\circ} \mathrm{C}$ for $6 \mathrm{~h}$. The cells were harvested by centrifugation at $5,000 \times g$ at $4^{\circ} \mathrm{C}$ for $30 \mathrm{~min}$, resuspended in $0.1 \mathrm{M} \mathrm{Tris}-\mathrm{HCl}$ buffer ( $\mathrm{pH} 8.0$ ), and disrupted by sonication at $4^{\circ} \mathrm{C}$ for $15 \mathrm{~min}$. Crude cell extracts were centrifuged at $15,000 \times g$ (at $4^{\circ} \mathrm{C}$ for $50 \mathrm{~min}$ ) to remove the cell debris. The resulting supernatant solution was placed on a histidine affinity column (HiTrap HP, GE Healthcare, USA) equilibrated with a $20 \mathrm{mM}$ Tris-Cl buffer (pH 8.0) in an LP system (Bio-Rad, USA). The rate of sample loading and column elution was maintained at $3.0 \mathrm{ml} / \mathrm{min}$ by the LP system. The recombinant proteins were eluted with a linear gradient of imidazole $(0-0.5 \mathrm{M})$ included in the same buffer, and active fractions were collected. Combined fractions were concentrated with Amicon Ultra- 15 Centrifugal Filter Units $(10,000 \mathrm{NMWL})\left(3,000 \times g\right.$ at $4^{\circ} \mathrm{C}$ for $\left.1-2 \mathrm{~h}\right)$ and stored at $4^{\circ} \mathrm{C}$ for further experimentation.

\section{Analysis of Non-Denaturing Polyacrylamide Gel Electrophoresis}

In total, $10 \mu \mathrm{M}$ each of purified mRFP1:AfuCoh76 and mRFP1:AfuDoc75 were combined in a 100- $\mu$ l mixture of $2 \mathrm{mM} \mathrm{CaCl}_{2}$ and Tris- $\mathrm{HCl}$ ( $\mathrm{pH}$ 8.0) for 30 min at room temperature for the formation of a complex between the AfuCoh76 and AfuDoc75 modules. Non-denaturing native polyacrylamide gel electrophoresis was performed using a $1.5-\mathrm{mm}$ thick $10 \%$ acrylamide resolving gel prepared in $125 \mathrm{mM}$ Tris- $\mathrm{HCl}(\mathrm{pH} 8.8)$. Samples were prepared in $62.5 \mathrm{mM}$ Tris- $\mathrm{HCl}(\mathrm{pH} 6.8)$ containing $10 \%(\mathrm{w} / \mathrm{v})$ glycerol and $0.01 \%(\mathrm{w} / \mathrm{v})$ bromophenol blue, but without 2-mercaptoethanol and SDS. The prepared samples were loaded on the $10 \%$ acrylamide gel without heating. Electrophoresis was performed in Tris-Glycine buffer ( $25 \mathrm{mM}$ Tris and $192 \mathrm{mM}$ glycine, $\mathrm{pH}$ 8.8) with 2 $\mathrm{mM} \mathrm{CaCl}_{2}$. Protein bands were stained for $30 \mathrm{~min}$ using $0.25 \%(\mathrm{w} / \mathrm{v})$ Coomassie brilliant blue and destained using a solution containing $20 \%(\mathrm{v} / \mathrm{v})$ methanol and $10 \%(\mathrm{v} / \mathrm{v})$ acetic acid.

\section{Display of AfuDoc75 and AfuCoh76 on the Surface of E. coli}

The resulting plasmids (pATLIC-AfuDoc75 and -AfuCoh76) were transformed into E. coli BW25113. The E. coli cells having the designated plasmids were cultured in $100 \mathrm{ml}$ of LB media supplemented with ampicillin $(100 \mu \mathrm{g} / \mathrm{ml})$ at $37^{\circ} \mathrm{C}$ and were induced at an $\mathrm{OD}_{600}$ of $0.6 \mathrm{by} \mathrm{L}(+)$-arabinose (final concentration of approximately $0.02 \%)$ at $16^{\circ} \mathrm{C}$ for $24 \mathrm{~h}$. Displayed cells were harvested by centrifugation $\left(3,000 \times g\right.$ at $4^{\circ} \mathrm{C}$ for $\left.10 \mathrm{~min}\right)$, and the cell pellet was washed with ice-cold $10 \mathrm{mM} \mathrm{NaCl}$ and stored at $-20^{\circ} \mathrm{C}$ for further experimentation.

\section{Verification of CSA Using mRFP1 Tagged with AfuCoh76 and AfuDoc75}

In total, $10 \mu \mathrm{M}$ of purified mRFP1:AfuDoc75 and mRFP1:AfuCoh76 was mixed with $2 \mathrm{ml}$ of culture (approximately $1 \times 10^{9}$ cells) of the cells displaying AfuDoc75 and AfuCoh76 at room temperature for $30 \mathrm{~min}$ in the presence of $2 \mathrm{mM} \mathrm{CaCl}_{2}$. After washing the unbound mRFP1 with PBS, the surface-bound mRFP1, bound by the interaction between the AfuCoh76 and AfuDoc75 modules, was measured using a Victor 3 spectrophotometer (Perkin-Elmer) with excitation at $590 \mathrm{~nm}$ ( $20 \mathrm{~nm}$ bandwidth) and emission at $616 \mathrm{~nm}(8.5 \mathrm{~nm}$ bandwidth $)$ in a $96-$ well plate. The background fluorescence of the cells was subtracted to obtain the relative fluorescence units.

\section{Detection of Whole Cell Activity for Surface-Attached Dimeric NABH in E. coli}

To efficiently purify proteins tagged with AfuDoc75, the crude cell extract containing overexpressed NABH (induction at $16^{\circ} \mathrm{C}$ for $24 \mathrm{~h}$ ) was directly mixed with $2 \mathrm{ml}$ of culture (approximately $1 \times 10^{9}$ cells) of the cells displaying AfuCoh76. The mixture was added to a final concentration of $2 \mathrm{~m} \mathrm{M} \mathrm{CaCl}_{2}$ and incubated for $30 \mathrm{~min}$ at room temperature. Enzyme-displaying cells were collected by centrifugation $\left(3,000 \times g\right.$ at $4^{\circ} \mathrm{C}$ for $\left.5 \mathrm{~min}\right)$ and incubated in $1 \mathrm{ml}$ reaction mixture $\left(2 \mathrm{mM} \mathrm{CaCl}_{2}\right.$ and $20 \mathrm{mM}$ Tris- $\left.\mathrm{HCl}, \mathrm{pH} 8.0\right)$ including $1.0 \%$ neoagarobiose (DP2, NAB) as the substrate of NABH at $25^{\circ} \mathrm{C}$ for $3 \mathrm{~h}$. The reaction products were analyzed by thin-layer chromatography (TLC) in a solvent system of $n$-butanol:ethanol:water $(3: 2: 2, \mathrm{v} / \mathrm{v})$ and visualized with $10 \%(\mathrm{v} / \mathrm{v})$ $\mathrm{H}_{2} \mathrm{SO}_{4}$ and $0.2 \%$ naphthoresorcinol in ethanol by heating [24].

\section{Results}

\section{Selection of Coh-Doc Pairs Among Various Bacteria}

Among 154 Coh-Doc pairs, we selected orthogonal Coh-Doc pairs that maintained specific binding between various POIs and anchoring scaffolds and that prevented cross-binding among Coh-Doc pairs. We evaluated the specificity and strength of bacterial Coh-Doc pairs based on the protein blot array image analysis [25] of the CohDoc pairs in the results reported by Haimovitz et al. [26]. 


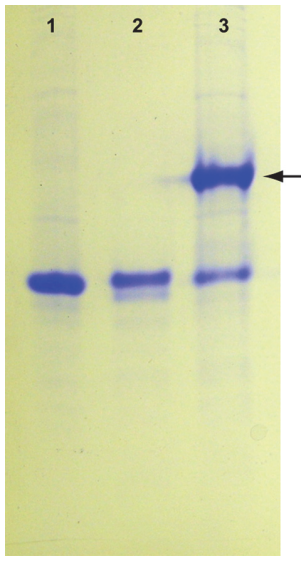

Fig. 1. Non-denaturing polyacrylamide gel electrophoresis analysis for the confirmation of complex formation of purified mRFP1:AfuCoh76 and mRFP1:AfuDoc75. Lane 1, purified mRFP1:AfuCoh76 (approximate M.W.: 39kDa, $20 \mu \mathrm{g}$ ); lane 2, purified mRFP1:AfuDoc75 (approximate M.W.: 33.4kDa, $20 \mu \mathrm{g}$ ); lane 3, the complex in a mixture of mRFP1:AfuCoh76 and mRFP1:AfuDoc75 (arrow).

Using all-against-all pairwise comparison, we identified the Coh-Doc pair of A. fulgidus as having the highest specificity and the strongest binding pair. The AfuCoh76-AfuDoc75 pair has previously been reported to exhibit strong and specific binding interactions [16]. Therefore, we chose the AfuCoh76-AfuDoc75 pair for the CSA system. The domain boundary was determined as previously reported [Doc domain in ORF2375 (432-506 amino acids) and Coh domain in ORF2376 (29-162 amino acids)] [16]. For heterologous expression of archaeal genes in E. coli, we optimized codons and synthesized AfuCoh76 and AfuDoc75 genes.

\section{Validation of In Vitro Binding Between mRFP1:AfuCoh76 and mRFP1:AfuDoc75 Modules}

Fusion proteins are generated by linking two proteins or domains of proteins by a peptide linker. The selection of a suitable linker sequence is of particular importance in the construction of functional fusion proteins [27]. Several studies related to recombinant Doc-containing proteins have not used any other special linker sequence because there are Doc domains linked to cellulases found in nature, which have a simple structure $[11,28]$. This feature offers an additional advantage when designing fusion proteins using a Doc domain as a tag. We confirmed the interaction between purified mRFP1 tagged with the AfuCoh76 or AfuDoc75 modules (mRFP1:AfuCoh76 or mRFP1:AfuDoc75, respectively) in vitro under non-denaturing conditions in the presence of $\mathrm{Ca}^{2+}$, which structurally maintains EF-hand coordination in the Coh and Doc modules (Fig. 1) [8]. This result indicates that each part can independently form a functional fold in fusion proteins without linker regions between heterologous proteins and the AfuDoc75 module.

Attachment of Heterologous Proteins on the Surface of $E$. coli

To verify functional attachment on the surface of $E$. coli, we combinatorially bound purified mRFP1:AfuCoh76 and mRFP1:AfuDoc75 to AfuCoh76- and AfuDoc75-displayed E. coli cells, respectively. As shown in Fig. 2, we only observed functional binding in the pairing between mRFP1:AfuDoc75 and AfuCoh76-diplayed E. coli cells. However, we failed to observe the red fluorescence of mRFP1:AfuCoh76 on the E. coli cells displaying AfuDoc75 for unknown reasons. We calculated the number of copies of mRFP1:AfuDoc75 bound to the cell surface

Cell surface displayed binding modules

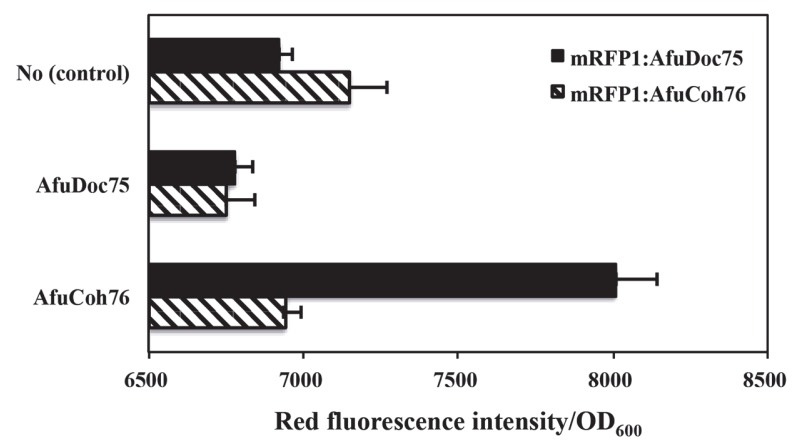

Fig. 2. Combinatorial binding tests of mRFP1:AfuCoh76 and mRFP1:AfuDoc75 to displayed AfuCoh76 and AfuDoc75. Only mRFP1:AfuDoc75 could be anchored to the surface-displayed AfuCoh76. 


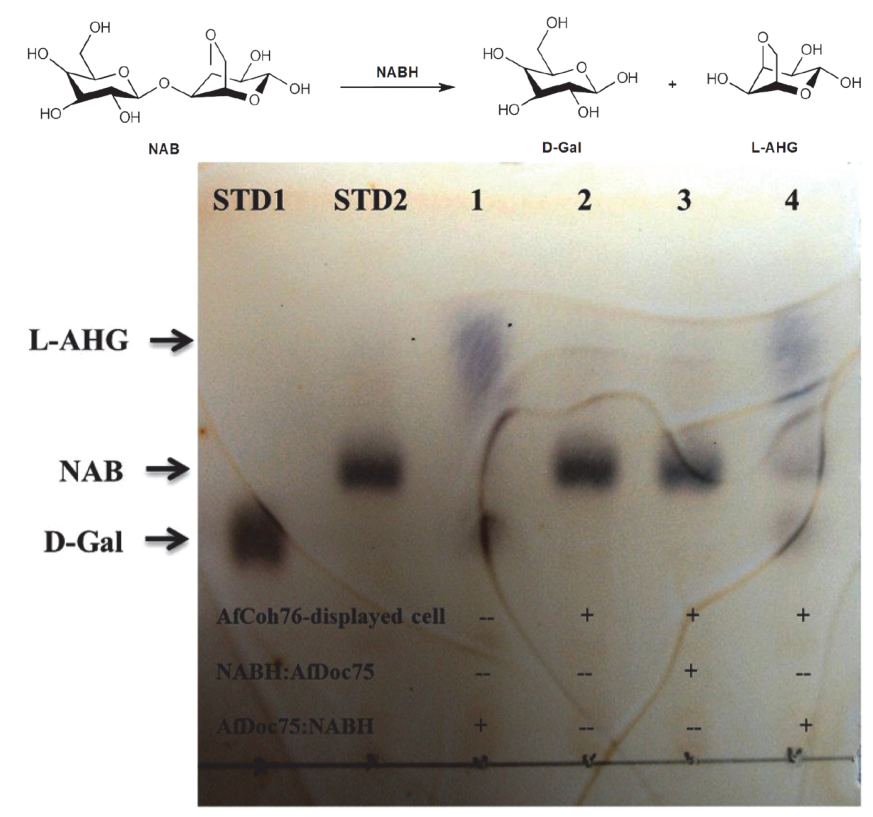

Fig. 3. Thin-layer chromatography analysis for the activity test of AfuDoc75:NABH-anchored cells. Lane STD1, D-galactose (D-Gal); STD2, neoagarobiose (NAB); 1, the reaction products in crude extract from overexpressed AfuDoc75:NABH (positive control); 2 , the reaction products from cells without displayed AfuCoh76; 3 , the reaction products from NABH:AfuDoc75-anchored cells (C-terminal fusion state); 4, the reaction products from AfuDoc75:NABH-anchored cells (N-terminal fusion state). D-Gal and 3,6-anhydro-L-galactose (L-AHG) are the reaction products degraded from NAB.

displaying AfuCoh76. Approximately 60,000 copies of mRFP1:AfuDoc75 were docked to the surface of cells, and this value corresponded to the number of copies of displayed mRFP1 reported in our previous study [17].

\section{CSA of NABH to the Surface of $E$. coli}

One of the current challenges in the surface display system is the expression of large multi-complex proteins [29]. Although several studies have shown that proteins with dimeric forms, such as bovine adrenodoxin and sorbitol dehydrogenase, can be actively displayed on the surface of $E$. coli, these studies were performed using an autodisplay platform with a high expression rate (e.g., AIDA-I autotransporter unit) $[5,6]$. The results from these studies did not completely overcome the structural limitation for the dimeric conformation but only partially overcame it. Because the established autodisplay platforms based on the type $\mathrm{V}$ secretion system are monomeric autotransporters [30], it is impossible to display multi-complex proteins using the currently available autodisplay platforms.

In a recent structural study on $\mathrm{NABH}$, the overall fold structure of the enzyme was found to be organized as an $\mathrm{N}$-terminal helical extension and a C-terminal, five-bladed $\beta$-propeller catalytic domain [21]. Because the Cterminus of NABH is the structurally critical position for dimerization in the active conformation, there is no CSD system currently available that can functionally display $\mathrm{NABH}$.

For the functional cell surface attachment of NABH on the surface of E. coli displaying AfuCoh76, we fused AfuDoc75 to the N-terminus of NABH (designated as AfuDoc75:NABH). The purified AfuDoc75:NABH was attached to the cell surface of $E$. coli displaying AfuCoh76. As expected, based on the attachment of the active dimeric form of $\mathrm{NABH}$ to the cell surface, we only detected the activity of NABH in a whole cell line anchoring AfuDoc75:NABH by a TLC chromatogram, and there was no NABH activity in any of the other whole cell lines (Fig. 3). This result indicates that the designed CSA system has the potential to attach multi-complex proteins using non-covalent interactions between Coh and Doc modules.

\section{Discussion}

A novel CSA system was designed using a non-covalent interaction to overcome the intrinsic limitations of the autodisplay platforms currently available. In the designed CSA system, it is possible to display multi-complex proteins, and there is no concern that disulfide bonds in displayed heterologous proteins may affect successful translocation to the outer membrane [4].

Although there are several modules present on bacterial cell surfaces that can mediate attachment via covalent or non-covalent interactions, such as S-layer homology domain [31], sortase-catalyzed cell wall attachment at LPXTG motif [32], choline-binding module [33], leucine-rich repeats [34], and lipoproteins [35], the designed CSA system that uses interactions between Coh and Doc domains offers several benefits. Because the AfuCoh76 and AfuDoc75 modules from A. fulgidus, in particular, exhibit the strongest recognition affinity, cross-linking to other cellular components can be effectively prevented. This system has the ability to tag the Doc module to the N- 
or C-terminus of anchored proteins without any linker sequence. It also has the potential for reversible specific binding by a $\mathrm{Ca}^{2+}$ switch by modifying the $\mathrm{Ca}^{2+}$-binding loop of the Doc module [36]. This system also offers the ability to directly decorate the Coh-displayed cells from crude cell extracts expressing Doc-fused proteins in a single step followed by simple purification steps.

In our previous study, although we failed to functionally display NABH through the YfaL autodisplay platform [17], we successfully and functionally anchored it to the surface of E. coli using AfuCoh76 and AfuDoc75 as biorecognition modules. The combination of the autodisplay platform and Coh-Doc module provides the opportunity to functionally display various multi-complex proteins on the surface of gram-negative bacteria.

\section{Acknowledgments}

This study was supported by a grant from Chosun University (2021).

\section{Conflict of Interest}

The authors have no financial conflicts of interest to declare.

\section{References}

1. van Bloois E, Winter RT, Kolmar H, Fraaije MW. 2011. Decorating microbes: surface display of proteins on Escherichia coli. Trends Biotechnol. 29: 79-86.

2. Lee SY, Choi JH, Xu Z. 2003. Microbial cell-surface display. Trends Biotechnol. 21: 45-52.

3. Rutherford N, Mourez M. 2006. Surface display of proteins by gram-negative bacterial autotransporters. Microb. Cell Fact. 5: 22 .

4. Nicolay T, Vanderleyden J, Spaepen S. 2015. Autotransporter-based cell surface display in Gram-negative bacteria. Crit. Rev. Microbiol. 41: 109-123.

5. Jose J, Bernhardt R, Hannemann F. 2002. Cellular surface display of dimeric Adx and whole cell P450-mediated steroid synthesis on E. coli. J. Biotechnol. 95: 257-268.

6. Jose J, von Schwichow S. 2004. Autodisplay of active sorbitol dehydrogenase (SDH) yields a whole cell biocatalyst for the synthesis of rare sugars. Chembiochem. 5: 491-499.

7. Bielen A, Teparic R, Vujaklija D, Mrsa V. 2014. Microbial anchoring systems for cell-surface display of lipolytic enzymes. Food Technol. Biotechnol. 52: 16 .

8. Bayer EA, Belaich JP, Shoham Y, Lamed R. 2004. The cellulosomes: multienzyme machines for degradation of plant cell wall polysaccharides. Annu. Rev. Microbiol. 58: 521-554

9. Chauvaux S, Beguin P, Aubert JP, Bhat KM, Gow LA, Wood TM, et al. 1990. Calcium-binding affinity and calcium-enhanced activity of Clostridium thermocellum endoglucanase D. Biochem. J. 265: 261-265.

10. Choi SK, Ljungdahl LG. 1996. Structural role of calcium for the organization of the cellulosome of Clostridium thermocellum. Biochemistry 35: 4906-4910.

11. Fierobe HP, Mechaly A, Tardif C, Belaich A, Lamed R, Shoham Y, et al. 2001. Design and production of active cellulosome chimeras. Selective incorporation of dockerin-containing enzymes into defined functional complexes. J. Biol. Chem. 276: 21257-21261.

12. Pagès S, Bélaïch A, Fierobe HP, Tardif C, Gaudin C, Bélaïch JP. 1999. Sequence analysis of scaffolding protein CipC and ORFXp, a new cohesin-containing protein in Clostridium cellulolyticum: comparison of various cohesin domains and subcellular localization of ORFXp. J. Bacteriol. 181: 1801-1810.

13. Pagès S, Belaich A, Tardif C, Reverbel-Leroy C, Gaudin C, Belaich JP. 1996. Interaction between the endoglucanase CelA and the scaffolding protein CipC of the Clostridium cellulolyticum cellulosome. J. Bacteriol. 178: 2279-2286.

14. Sakka K, Sugihara Y, Jindou S, Sakka M, Inagaki M, Sakka K, et al. 2011. Analysis of cohesin-dockerin interactions using mutant dockerin proteins. FEMS Microbiol. Lett. 314: 75-80.

15. Salama-Alber O, Jobby MK, Chitayat S, Smith SP, White BA, Shimon LJW, et al. 2013. Atypical cohesin-dockerin complex responsible for cell surface attachment of cellulosomal components: binding fidelity, promiscuity, and structural buttresses. J. Biol. Chem. 288: 16827-16838.

16. Voronov-Goldman M, Lamed R, Noach I, Borovok I, Kwiat M, Rosenheck S, et al. 2011. Noncellulosomal cohesin from the hyperthermophilic archaeon Archaeoglobus fulgidus. Proteins 79: 50-60.

17. Ko HJ, Park E, Song J, Yang TH, Lee HJ, Kim KH, et al. 2012. Functional cell surface display and controlled secretion of diverse Agarolytic enzymes by Escherichia coli with a novel ligation-independent cloning vector based on the autotransporter YfaL. Appl. Environ. Microbiol. 78: 3051-3058.

18. Chung CT, Niemela SL, Miller RH. 1989. One-step preparation of competent Escherichia coli: transformation and storage of bacterial cells in the same solution. Proc. Natl. Acad. Sci. USA 86: 2172-2175.

19. Ekborg NA, Taylor LE, Longmire AG, Henrissat B, Weiner RM, Hutcheson SW. 2006. Genomic and proteomic analyses of the agarolytic system expressed by Saccharophagus degradans 2-40. Appl. Environ. Microbiol. 72: 3396-3405.

20. Campbell RE, Tour O, Palmer AE, Steinbach PA, Baird GS, Zacharias DA, et al. 2002. A monomeric red fluorescent protein. Proc. Natl. Acad. Sci. USA 99: 7877-7882.

21. Ha SC, Lee S, Lee J, Kim HT, Ko HJ, Kim KH, et al. 2011. Crystal structure of a key enzyme in the agarolytic pathway, a-neoagarobiose hydrolase from Saccharophagus degradans 2-40. Biochem. Biophys. Res. Commun. 412: 238-244.

22. Nelson MD, Fitch DH. 2011. Overlap extension PCR: an efficient method for transgene construction. Methods Mol. Biol. 772: 459-470.

23. Lee J, Kim SH. 2009. High-throughput T7 LIC vector for introducing C-terminal poly-histidine tags with variable lengths without extra sequences. Protein Expr. Purif. 63: 58-61.

24. Duckworth M, Yaphe W. 1970. Thin-layer chromatographic analysis of enzymic hydrolysates of agar. J. Chromatogr. 49: $482-487$.

25. Schneider CA, Rasband WS, Eliceiri KW. 2012. NIH Image to ImageJ: 25 years of image analysis. Nat. Methods 9: 671-675.

26. Haimovitz R, Barak Y, Morag E, Voronov-Goldman M, Shoham Y, Lamed R, et al. 2008. Cohesin-dockerin microarray: Diverse specificities between two complementary families of interacting protein modules. Proteomics 8: 968-979.

27. Arai R, Ueda H, Kitayama A, Kamiya N, Nagamune T. 2001. Design of the linkers which effectively separate domains of a bifunctional fusion protein. Protein Eng. 14: 529-532.

28. Caspi J, Barak Y, Haimovitz R, Irwin D, Lamed R, Wilson DB, et al. 2009. Effect of linker length and dockerin position on conversion of a Thermobifida fusca endoglucanase to the cellulosomal mode. Appl. Environ. Microbiol. 75: 7335-7342.

29. Wu CH, Mulchandani A, Chen W. 2008. Versatile microbial surface-display for environmental remediation and biofuels production. Trends Microbiol. 16: 181-188.

30. Leo JC, Grin I, Linke D. 2012. Type V secretion: mechanism(s) of autotransport through the bacterial outer membrane. Philos. Trans. R Soc. Lond B Biol. Sci. 367: 1088-1101. 
31. Zarschler K, Janesch B, Kainz B, Ristl R, Messner P, Schäffer C. 2010. Cell surface display of chimeric glycoproteins via the S-layer of Paenibacillus alvei. Carbohydr. Res. 345: 1422-1431.

32. Liew PX, Wang CL, Wong SL. 2012. Functional characterization and localization of a Bacillus subtilis sortase and its substrate and use of this sortase system to covalently anchor a heterologous protein to the B. subtilis cell wall for surface display. J. Bacteriol. 194: 161-175.

33. Bello-Gil D, Maestro B, Fonseca J, Feliu JM, Climent V, Sanz JM. 2014. Specific and reversible immobilization of proteins tagged to the affinity polypeptide C-LytA on functionalized graphite electrodes. PLoS One 9: e87995.

34. Loimaranta V, Hytönen J, Pulliainen AT, Sharma A, Tenovuo J, Strömberg N, et al. 2009. Leucine-rich repeats of bacterial surface proteins serve as common pattern recognition motifs of human scavenger receptor gp340. J. Biol. Chem. 284: 18614-18623.

35. Berlec A, Zadravec P, Jevnikar Z, Štrukelj B. 2011. Identification of candidate carrier proteins for surface display on Lactococcus lactis by theoretical and experimental analyses of the surface proteome. Appl. Environ. Microbiol. 77: 1292-1300.

36. Karpol A, Kantorovich L, Demishtein A, Barak Y, Morag E, Lamed R, et al. 2009. Engineering a reversible, high-affinity system for efficient protein purification based on the cohesin-dockerin interaction. J. Mol. Recognit. 22: 91-98. 